\title{
Nonlinear Dynamics and Chaos in a Fractional-Order HIV Model
}

\author{
Haiping Ye ${ }^{1,2}$ and Yongsheng Ding ${ }^{2,3}$ \\ ${ }^{1}$ Department of Applied Mathematics, Donghua University, Shanghai 201620, China \\ ${ }^{2}$ College of Information Sciences and Technology, Donghua University, Shanghai 201620, China \\ ${ }^{3}$ Engineering Research Center of Digitized Textile and Fashion Technology, Ministry of Education, \\ Donghua University, Shanghai 201620, China
}

Correspondence should be addressed to Yongsheng Ding, ysding@dhu.edu.cn

Received 18 January 2009; Revised 26 February 2009; Accepted 8 April 2009

Recommended by José Roberto Castilho Piqueira

We introduce fractional order into an HIV model. We consider the effect of viral diversity on the human immune system with frequency dependent rate of proliferation of cytotoxic T-lymphocytes (CTLs) and rate of elimination of infected cells by CTLs, based on a fractional-order differential equation model. For the one-virus model, our analysis shows that the interior equilibrium which is unstable in the classical integer-order model can become asymptotically stable in our fractionalorder model and numerical simulations confirm this. We also present simulation results of the chaotic behaviors produced from the fractional-order HIV model with viral diversity by using an Adams-type predictor-corrector method.

Copyright (C) 2009 H. Ye and Y. Ding. This is an open access article distributed under the Creative Commons Attribution License, which permits unrestricted use, distribution, and reproduction in any medium, provided the original work is properly cited.

\section{Introduction}

An important part of the human immune response against viral infections is cytotoxic $\mathrm{T}$ lymphocytes (CTLs) [1]. They recognize and kill cells which are infected by virus. There are many immune models describing the virus dynamics with CTL immune response. Nowak and Bangham $[2,3]$ proposed an ODE model which explores the relation among CTL immune responses, virus load, and virus diversity. In [2], a rate of specific CTL $\left(Z_{j}\right)$ proliferation in response to the corresponding specific infected cells $\left(I_{j}\right)$ depends on the mass action law $c I_{j} Z_{j}$. This model has been important in the field of mathematical modelling of HIV infection. In their model, there is no interaction among different types of CTL $\left(Z_{j}\right)$. Iwami et al. [4] assumed that the correlation is incorporated as a function of the frequency that the specific CTLs $\left(Z_{j}\right)$ encounter in the specific infected cells $\left(I_{j}\right)$. In a similar manner, they considered the rate of elimination of specific infected cells $\left(I_{j}\right)$ by the specific CTLs $\left(Z_{j}\right)$ to be proportional to this frequency. However, these models do not take into account 
the fractional order derivatives that have been extensively applied in many fields (e.g., [517] and the reference cited therein). Recently many mathematicians and applied researchers have tried to model real processes using the fractional order differential equations (FODE) [16]. In biology, it has been deduced that the membranes of cells of biological organism have fractional order electrical conductance [13] and then, they are classified into group of noninteger order models. Also, it has been shown that modelling the behavior of brainstem vestibule-oculumotor neurons by FODE has more advantages than classical integer order modelling [8].

Particular emphasis is that a major difference between fractional order models and integer order models is that fractional order models possess memory $[5,12]$, while the main features of immune response involve memory [18]. Hence, we attempt to model HIV infection with immune response using a fractional order system. Our presentation is based on the immune model of HIV infection which is developed by Iwami et al. [4]. For the one-virus model, we carry out a detailed analysis on stability of equilibrium. Our analysis shows that the interior equilibrium which is unstable in the classical integer order model can become asymptotically stable in our fractional order model. We also find that chaos does exist in the fractional order HIV model with viral diversity.

\section{Model Derivation}

We first give the definition of fractional order integration and fractional order differentiation $[14,16]$. For the concept of fractional derivative we will adopt Caputo's definition which is a modification of the Riemann-Liouville definition and has the advantage of dealing properly with initial value problems.

Definition 2.1. The fractional integral of order $\alpha>0$ of a function $f: R^{+} \rightarrow R$ is given by

$$
I^{\alpha} f(x)=\frac{1}{\Gamma(\alpha)} \int_{0}^{x}(x-t)^{\alpha-1} f(t) d t
$$

provided the right side is pointwise defined on $R^{+}$.

Definition 2.2. The Caputo fractional derivative of order $\alpha \in(n-1, n)$ of a continuous function $f: R^{+} \rightarrow R$ is given by

$$
D^{\alpha} f(x)=I^{n-\alpha} D^{n} f(x), \quad D=\frac{d}{d t} .
$$

Now we introduce fractional order into the ODE model by Iwami et al. [4]. The new system is described by the following set of FODE:

$$
\begin{gathered}
D^{q_{1}} T=\lambda-d T-\sum_{l=1}^{n} \beta_{l}^{\prime} T V_{l}, \\
D^{q_{2 j}} I_{j}=\beta_{j}^{\prime} T V_{j}-a I_{j}-b Z_{j} \frac{I_{j}}{T+\sum_{l=1}^{n} I_{l}},
\end{gathered}
$$




$$
\begin{gathered}
D^{q_{3 j}} Z_{j}=c Z_{j} \frac{I_{j}}{T+\sum_{l=1}^{n} I_{l}}-\delta Z_{j}, \\
D^{q_{4 j}} V_{j}=k a I_{j}-u V_{j} \quad(j=1,2, \ldots, n),
\end{gathered}
$$

where $T(t)$ represents the concentration of uninfected cells at time $t, I_{j}(t)$ represents the concentration of infected cells with a virus particle of type $j, V_{j}(t)$ the concentration of free virus particle of type $j$, and $Z_{j}(t)$ denotes the magnitude of the specific CTL response against variant $j$. Here, $0.95 \leq q_{1}, q_{2 j}, q_{3 j}, q_{4 j} \leq 1(j=1,2, \ldots, n)$ are restricted such that fractional derivative can be approximately described the rate of change in number.

Following [4], uninfected cells are assumed to be generated at a constant rate $\lambda$. Uninfected cells, infected cells, free viruses, and CTLs decline at rates $d, a, u$, and $\delta$, respectively. The total number of virus particles produced from one cell is $k$. The rate of CTL proliferation in response to antigen is given by $c Z_{j} I_{j} /\left(T+\sum_{l=1}^{n} I_{l}\right)$ and the specific infected cells are killed by specific CTLs at rate $b Z_{j} I_{j} /\left(T+\sum_{l=1}^{n} I_{l}\right)$, while infected cells are produced from uninfected cells and free virus at rate $\beta_{l}^{\prime} T V_{l}$. That is, a rate of specific $\operatorname{CTL}\left(Z_{j}\right)$ proliferation in response to the corresponding specific infected cells $\left(I_{j}\right)$ depends on the frequency, instead of the mass action law.

To simplify the model, it is reasonable to assume that the decay rate of free virus, $u$, is much larger than that of the infected cells, $a$, and this system describes the qualitative dynamics of the asymptomatic phase of HIV infection. Thus, we may introduce as a good approximation that the virus is in steady state (i.e., $D^{q_{4 j}} V_{j}=0$ ) and hence $V_{j}=k a I_{j} / u$ (see $[4,19])$. This leads to the following simplified system of FODE:

$$
\begin{gathered}
D^{q_{1}} T=\lambda-d T-\sum_{l=1}^{n} \beta_{l} T I_{l}, \\
D^{q_{2 j}} I_{j}=\beta_{j} T I_{j}-a I_{j}-b Z_{j} \frac{I_{j}}{T+\sum_{l=1}^{n} I_{l}}, \\
D^{q_{3 j}} Z_{j}=c Z_{j} \frac{I_{j}}{T+\sum_{l=1}^{n} I_{l}}-\delta Z_{j} \quad(j=1,2, \ldots, n),
\end{gathered}
$$

where $\beta_{j}=k a \beta_{j}^{\prime} / u$.

\section{One-Virus Model}

In this section, we discuss in detail an important special case of model (2.4) and perform an equilibrium and stability analysis for this special case. We consider the one-virus model $(n=1)$ and assume that $q_{1}=q_{21}=q_{31}=\alpha(0.95 \leq \alpha \leq 1)$. This one-virus model is described by the following system of FODE:

$$
\begin{gathered}
D^{\alpha} T=\lambda-d T-\beta_{1} T I_{1}, \\
D^{\alpha} I_{1}=\beta_{1} T I_{1}-a I_{1}-\frac{b Z_{1} I_{1}}{T+I_{1}}, \\
D^{\alpha} Z_{1}=\frac{c Z_{1} I_{1}}{T+I_{1}}-\delta Z_{1} .
\end{gathered}
$$


To evaluate the equilibria, let

$$
\begin{gathered}
D^{\alpha} T=0, \\
D^{\alpha} I_{1}=0, \\
D^{\alpha} Z_{1}=0 .
\end{gathered}
$$

Then system (3.1) has three equilibria: the uninfected equilibrium $E_{H}=(\lambda / d, 0,0)$, the boundary equilibrium $E_{I}=\left(T^{*}, I_{1}^{*}, 0\right)$, where

$$
T^{*}=\frac{a}{\beta_{1}}, \quad I_{1}^{*}=\frac{\lambda}{a}-\frac{d}{\beta_{1}},
$$

and the interior equilibrium $E_{c}=\left(\widehat{T}, \widehat{I}_{1}, \widehat{Z}_{1}\right)$, where

$$
\begin{gathered}
\widehat{T}=\frac{-d+\sqrt{d^{2}+4 \lambda \hat{\beta}}}{2 \widehat{\beta}}, \quad \widehat{I}_{1}=\frac{\delta}{c-\delta} \widehat{T}, \\
\widehat{Z}_{1}=\frac{c \widehat{T}}{b(c-\delta)}\left(\beta_{1} \widehat{T}-a\right), \quad \widehat{\beta}=\frac{\delta \beta_{1}}{c-\delta} .
\end{gathered}
$$

Following the analysis in [4], we introduce a basic reproduction number which is defined by

$$
R_{0}=\frac{\lambda \beta_{1}}{a d}
$$

Denote $R_{+}^{3}=\left\{\left(T, I_{1}, Z_{1}\right) \in R^{3} \mid T \geq 0, I_{1} \geq 0, Z_{1} \geq 0\right\}$ and we always assume that $c>\delta$. Note that $D^{\alpha} Z_{1}<0$ always holds true if $c \leq \delta$. By generalized mean value theorem [15], we get $Z_{1}(t)$ is decreasing if $c \leq \delta$.

Next we will discuss the existence and stability of the equilibria of the model (3.1).

Theorem 3.1. (a) The uninfected equilibrium $E_{H}$ is locally asymptotically stable (LAS) if $R_{0} \in(0,1)$ and unstable if $R_{0}>1$.

(b) If $R_{0}>1$, then the boundary equilibrium $E_{I}$ exists. This equilibrium is LAS if $1<R_{0}<$ $(a \delta / d(c-\delta))+1$ and unstable if $R_{0}>(a \delta / d(c-\delta))+1$.

(c) If $R_{0}>(a \delta / d(c-\delta))+1$, then $E_{c}$ exists in $\operatorname{Int} R_{+}^{3}$, where $\operatorname{Int} R_{+}^{3}$ is the interior of $R_{+}^{3}$.

Proof. (a) The Jacobian matrix $J\left(E_{H}\right)$ for system (3.1) evaluated at $E_{H}$ is given by

$$
J\left(E_{H}\right)=\left(\begin{array}{ccc}
-d & -\frac{\beta_{1} \lambda}{d} & 0 \\
0 & \frac{\beta_{1} \lambda}{d}-a & 0 \\
0 & 0 & -\delta
\end{array}\right) .
$$


$E_{H}$ is locally asymptotically stable if all of the eigenvalues $p$ of the Jacobian matrix $J\left(E_{H}\right)$ satisfy the following condition $[6,17]$ :

$$
|\arg (p)|>\frac{\alpha \pi}{2}
$$

The eigenvalues of $J\left(E_{H}\right)$ are $-d, \beta_{1} \lambda / d-a,-\delta$. It is clear that $E_{H}$ is LAS if $R_{0}<1$ and is unstable if $R_{0}>1$.

(b) If $R_{0}>1$, then the existence of $E_{I}$ is obvious.

The Jacobian matrix $J\left(E_{I}\right)$ for system (3.1) evaluated at $E_{I}$ is given by

$$
J\left(E_{I}\right)=\left(\begin{array}{ccc}
-d-\beta_{1} I_{1}^{*} & -\beta_{1} T^{*} & 0 \\
\beta_{1} I_{1}^{*} & 0 & -\frac{b I_{1}^{*}}{T^{*}+I_{1}^{*}} \\
0 & 0 & \frac{c I_{1}^{*}}{T^{*}+I_{1}^{*}}-\delta
\end{array}\right)
$$

For $J\left(E_{I}\right)$ given by (3.8), the characteristic equation becomes

$$
\left[p^{2}+\left(d+\beta_{1} I_{1}^{*}\right) p+\beta_{1}^{2} I_{1}^{*} T^{*}\right]\left(p-\frac{c I_{1}^{*}}{T^{*}+I_{1}^{*}}+\delta\right)=0 .
$$

and hence all the eigenvalues are

$$
\begin{gathered}
p_{1,2}=\frac{-\left(d+\beta_{1} I_{1}^{*}\right) \pm \sqrt{\left(d+\beta_{1} I_{1}^{*}\right)^{2}-4 \beta_{1}^{2} I_{1}^{*} T^{*}}}{2}, \\
p_{3}=\frac{c I_{1}^{*}}{T^{*}+I_{1}^{*}}-d .
\end{gathered}
$$

If $R_{0}>1$, then $T^{*}>0, I_{1}^{*}>0$, and $p_{1,2}$ have negative real parts. Furthermore, if $1<R_{0}<$ $(a \delta / d(c-\delta))+1$, then $p_{3}<0$ and $E_{I}$ is LAS. If $R_{0}>(a \delta / d(c-\delta))+1$, then $p_{3}>0$ and $E_{I}$ is unstable.

(c) If $R_{0}>(a \delta / d(c-\delta))+1$, then we obtain $\widehat{Z}_{1}>0$. Thus, $E_{c}$ exists in Int $R_{+}^{3}$. Therefore, the proof is complete.

To discuss the local stability of the interior equilibrium $E_{c}$, we consider the linearized system of (3.1) at $E_{c}$. The Jacobian matrix at $E_{c}$ is given by

$$
J\left(E_{c}\right)=\left(\begin{array}{ccc}
-d-\beta_{1} \widehat{I}_{1} & -\beta_{1} \widehat{T} & 0 \\
\beta_{1} \widehat{I}_{1}+\frac{b \widehat{Z}_{1} \widehat{I}_{1}}{\left(\widehat{T}+\widehat{I}_{1}\right)^{2}} & \beta_{1} \widehat{T}-a-\frac{b \widehat{Z}_{1} \widehat{T}}{\left(\widehat{T}+\widehat{I}_{1}\right)^{2}} & -\frac{b \widehat{I}_{1}}{\widehat{T}+\widehat{I}_{1}} \\
-\frac{c \widehat{Z}_{1} \widehat{I}_{1}}{\left(\widehat{T}+\widehat{I}_{1}\right)^{2}} & \frac{c \widehat{Z}_{1} \widehat{T}}{\left(\widehat{T}+\widehat{I}_{1}\right)^{2}} & \frac{c \widehat{I}_{1}}{\widehat{T}+\widehat{I}_{1}}-\delta
\end{array}\right) .
$$


For convenience, we denote $\widehat{T}=T, \widehat{I}_{1}=I, \widehat{Z}_{1}=Z$, and $\beta_{1}=\beta$. In view of the above assumptions and using

$$
\beta T-a-\frac{b Z}{T+I}=0, \quad \lambda-d T-\beta T I=0, \quad \frac{c I}{T+I}=\delta, \quad \frac{T}{T+I}=\frac{c-\delta}{c},
$$

$J\left(E_{c}\right)$ can now be written as follows:

$$
J\left(E_{c}\right)=\left(\begin{array}{ccc}
-d-\beta I & -\beta T & 0 \\
\beta I+\frac{\delta}{c}(\beta T-a) & \frac{\delta}{c}(\beta T-a) & -\frac{b \delta}{c} \\
-\frac{\delta}{b}(\beta T-a) & \frac{c-\delta}{b}(\beta T-a) & 0
\end{array}\right) .
$$

Then the characteristic equation of the linearized system of (3.1) is

$$
\Phi(p)=p^{3}+a_{1} p^{2}+a_{2} p+a_{3}=0
$$

where

$$
\begin{gathered}
a_{1}=d+\frac{\delta a}{c}+\frac{\delta^{2} \beta T}{c(c-\delta)}, \\
a_{2}=\beta T\left(\beta I+\frac{\delta}{c}(\beta T-a)\right)+\frac{\delta}{c}(c-\delta)(\beta T-a)-\frac{\delta}{c}(d+\beta I)(\beta T-a), \\
a_{3}=\frac{\delta}{c}(c-\delta)(\beta T-a)(d+\beta I)+\frac{\beta \delta^{2}}{c} T(\beta T-a) .
\end{gathered}
$$

Proposition 3.2. The interior equilibrium $E_{c}$ is LAS if all of the eigenvalues $p$ of $J\left(E_{c}\right)$ satisfy $|\arg (p)|>\alpha \pi / 2$.

Denote

$$
\begin{aligned}
D(\Phi) & =-\left|\begin{array}{ccccc}
1 & a_{1} & a_{2} & a_{3} & 0 \\
0 & 1 & a_{1} & a_{2} & a_{3} \\
3 & 2 a_{1} & a_{2} & 0 & 0 \\
0 & 3 & 2 a_{1} & a_{2} & 0 \\
0 & 0 & 3 & 2 a_{1} & a_{2}
\end{array}\right| \\
& =18 a_{1} a_{2} a_{3}+\left(a_{1} a_{2}\right)^{2}-4 a_{3} a_{1}^{3}-4 a_{2}^{3}-27 a_{3}^{2} .
\end{aligned}
$$




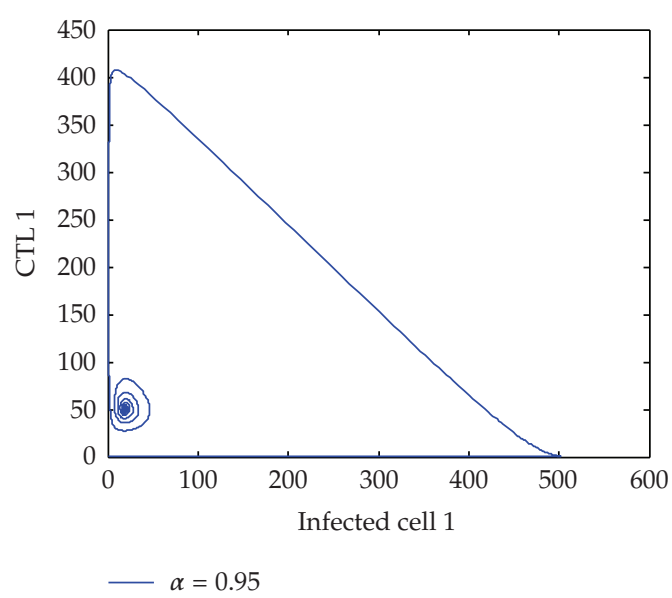

(a)

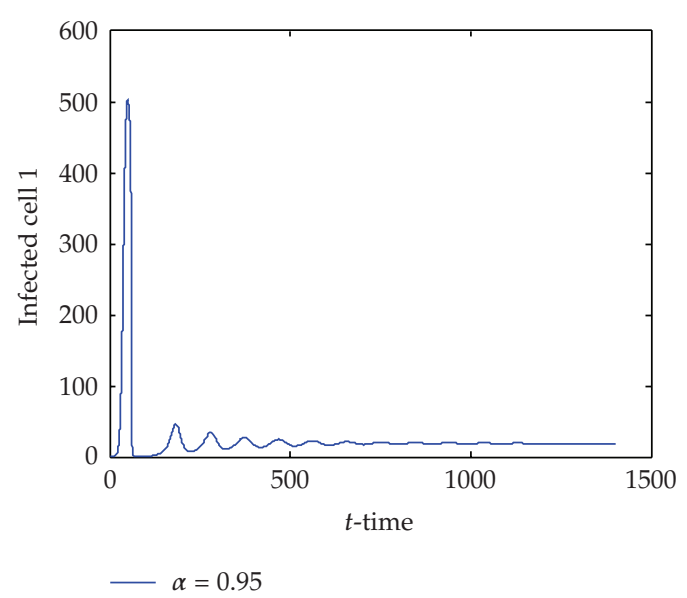

(b)

Figure 1: Numerical solutions of system (3.1). The plots show that trajectories of system (3.1) approach to the interior equilibrium for $\alpha=0.95$.

Using the results of $[5,20]$, we have the following proposition.

Proposition 3.3. One assumes that $E_{c}$ exists in $\operatorname{Int} R_{+}^{3}$.

(i) If the discriminant of $\Phi(p), D(\Phi)$ is positive and Routh-Hurwitz conditions are satisfied, that is,

$$
D(\Phi)>0, \quad a_{1}>0, \quad a_{3}>0, \quad a_{1} a_{2}>a_{3},
$$

then the interior equilibrium $E_{c}$ is LAS.

(ii) If $D(\Phi)<0, a_{1}>0, a_{2}>0, a_{1} a_{2}=a_{3}, \alpha \in[0,1)$, then the interior equilibrium $E_{c}$ is LAS.

(iii) If $D(\Phi)<0, a_{1}<0, a_{2}<0, \alpha>2 / 3$, then the interior equilibrium $E_{c}$ is unstable.

In our first example we set $\lambda=10, d=0.02$ which are chosen according to [21] and set $a=\delta=0.04, c=b=0.8, \beta_{1}=4 \times 10^{-4}$ which come from [4]. With these parameter values, $R_{0}=5>(a \delta / d(c-\delta))+1, D(\Phi)=-9.4073 \times 10^{-7}<0, a_{1} a_{2}<a_{3}$. By Proposition 3.2., we obtain the interior equilibrium $E_{c}=(362.0335,19.0544,49.9289)$ is LAS when $\alpha<0.9916$. Numerical simulations show that trajectories of system (3.1) approach to the interior equilibrium (see Figures 1(a) and 1(b)). However, when $\alpha=1$ (that is the case of classical integer order), $E_{c}$ is unstable by the Routh-Hurwitz criterion(see Figures 2(a) and 2(b)).

\section{Two-Virus Model}

In this section, we consider viral diversity. We examine the two-virus model using numerical simulations. By examining the behavior of this simpler model we hope to get an idea as to 


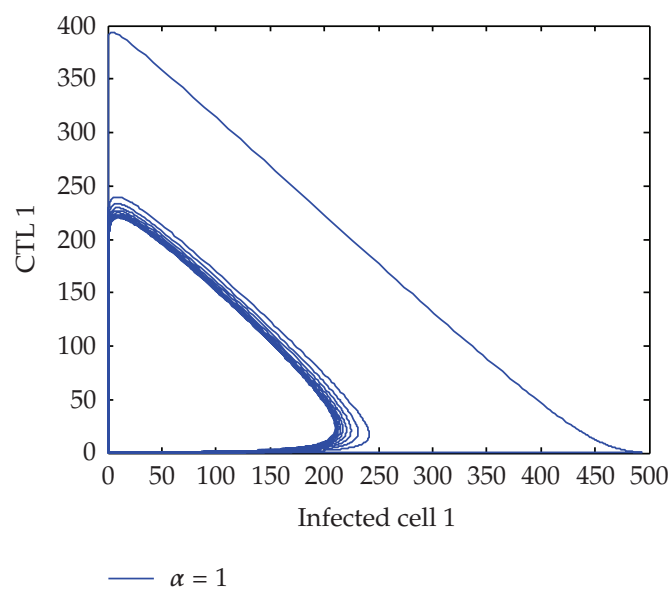

(a)

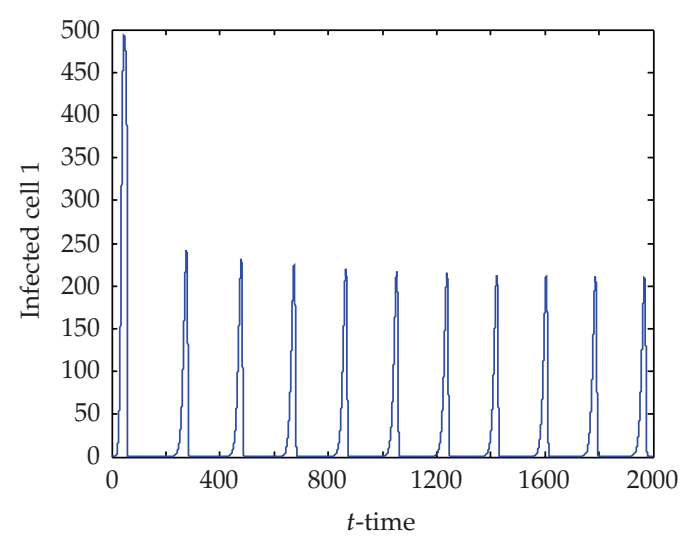

(b)

Figure 2: Numerical solutions of system (3.1). The plots show that the interior equilibrium is unstable for $\alpha=1$.

how the more general models in system (2.4) may behave. The two-virus model is given by the following system of FODE:

$$
\begin{gathered}
D^{q_{1}} T=\lambda-d T-\beta_{1} T I_{1}-\beta_{2} T I_{2}, \\
D^{q_{21}} I_{1}=\beta_{1} T I_{1}-a I_{1}-b Z_{1} \frac{I_{1}}{T+I_{1}+I_{2}}, \\
D^{q_{22}} I_{2}=\beta_{2} T I_{2}-a I_{2}-b Z_{2} \frac{I_{2}}{T+I_{1}+I_{2}}, \\
D^{q_{31}} Z_{1}=c Z_{1} \frac{I_{1}}{T+I_{1}+I_{2}}-\delta Z_{1}, \\
D^{q_{32}} Z_{2}=c Z_{2} \frac{I_{2}}{T+I_{1}+I_{2}}-\delta Z_{2},
\end{gathered}
$$

with initial value condition

$$
T(0)=T_{0}, \quad I_{i}(0)=I_{i 0}, \quad Z_{i}(0)=Z_{i 0}, \quad i=1,2,
$$

where $0.95 \leq q_{1}, q_{2 j}, q_{3 j}(j=1,2) \leq 1$.

To find numerical solution to (4.1) and (4.2) in the interval $[0, T]$, we reduce the systems (4.1) and (4.2) to a set of fractional integral equations, by using an equivalence (see [16, Theorem 3.24])

$$
D^{\alpha} X=f(X) \Longleftrightarrow X(t)=X(0)+I^{\alpha} f(X)
$$

Then we apply the generalized Adams-type predictor-corrector method or, more precisely, Predict, Evaluate, Correct, Evaluate (PECE) methods (see [22, 23]). 


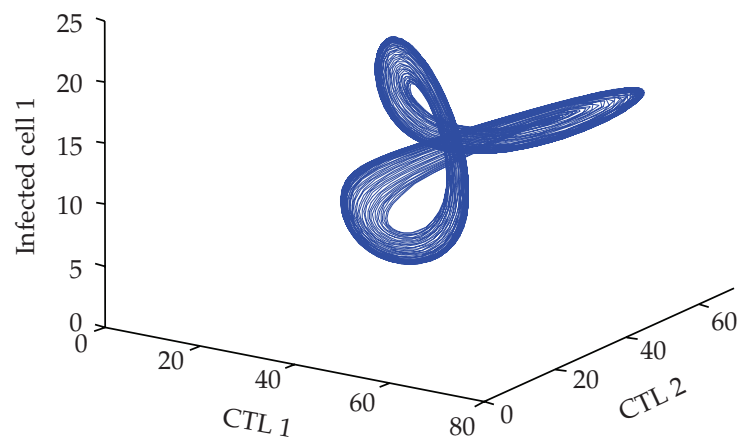

(a)

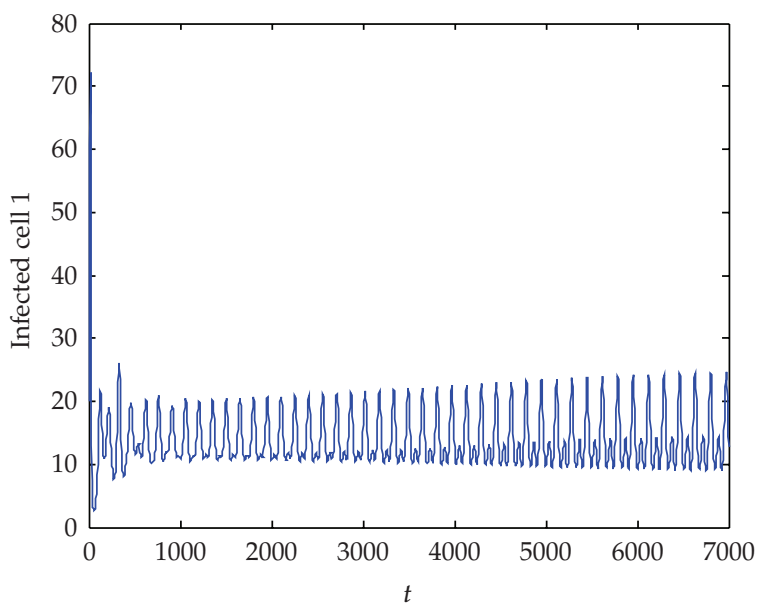

(b)

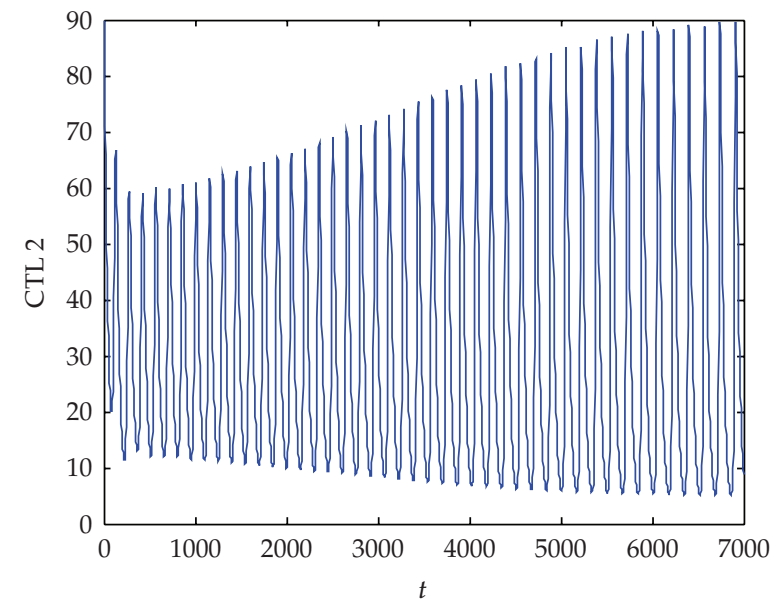

(c)

Figure 3: Numerical solutions of system (4.1) for $\alpha=[0.95,0.95,1,1,1]$. (a) A strange attractor in the $Z_{1}-Z_{2}-I_{1}$ phase. (b) Infected cell 1. (c) CTL 2. 


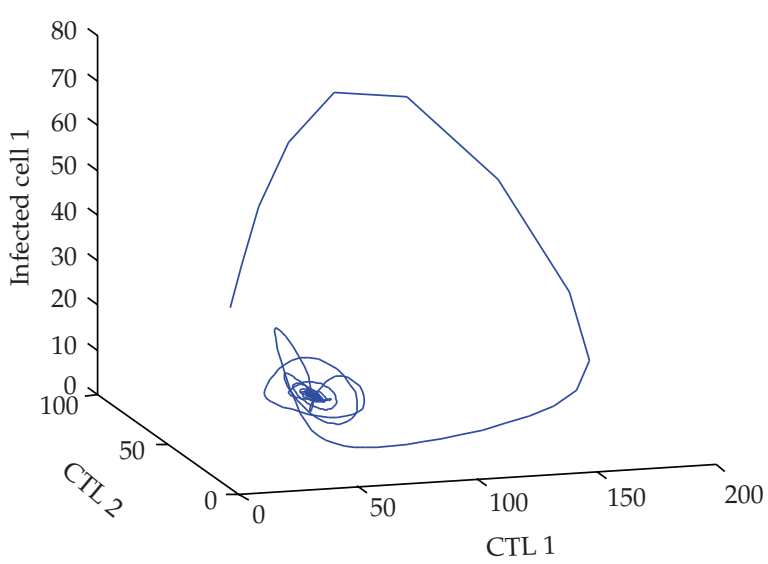

(a)

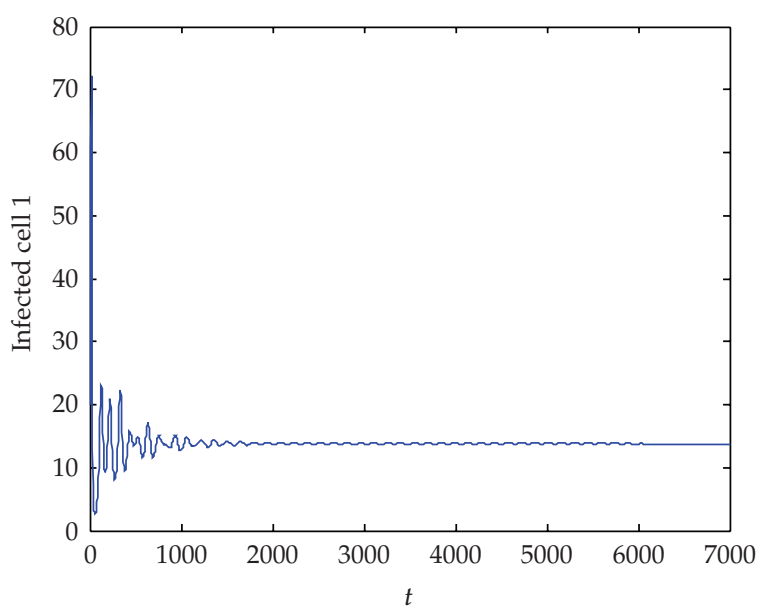

(b)

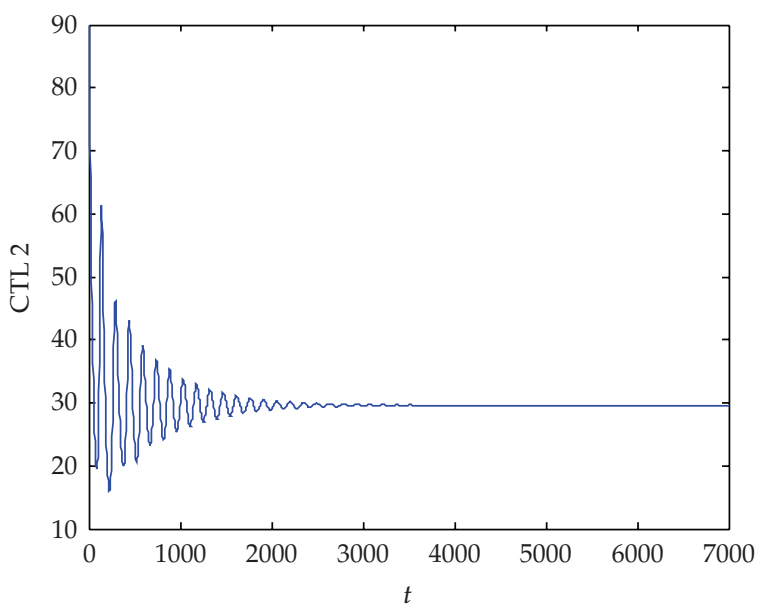

(c)

Figure 4: Numerical solutions of system (4.1) for $\alpha=[0.95,0.95,0.95,1,1]$. (a) $Z_{1}-Z_{2}-I_{1}$ phase. (b) Infected cell 1. (c) CTL 2. 
For notational convenience, we denote $\alpha=\left(q_{1}, q_{21}, q_{22}, q_{31}, q_{32}\right)$. We carry out numerical simulations for system, (4.1) and (4.2) with parameters $\lambda=10, b=c=0.8, a=0.031, \delta=$ $0.03, d=0.02, \beta_{1}=4 \times 10^{-4}$, and $\beta_{2}=2.8 \times 10^{-4}$ for the step size 0.07 . Numerical solutions of systems (4.1) and (4.2) support that the system exhibits a chaotic behavior and systems (4.1) and (4.2) have a strange attractor in Int $R_{+}^{5}$ for $\alpha=[0.95,0.95,1,1,1]$ (see Figures 3(a)$3(\mathrm{c}))$. It is clear that chaos does exist in our fractional order model with viral diversity as in the case of integer order model. The effect of viral diversity and the frequency dependence results in collapse of the immune system and make the behavior of the system dynamics complex [4]. However, as the value of some component or more components of the order $\alpha$ further decreases, for example, $\alpha=[0.95,0.95,0.95,1,1]$, the chaotic motion disappears and the systems (4.1) and (4.2) stabilize to a fixed point (see Figures 4(a) $-4(\mathrm{c})$ ).

\section{Conclusions}

In this paper, we have proposed a fractional order HIV model, as a generalization of an integer order model, developed by Iwami et al. [4]. The premise of the proposed model is the fact that fractional order models possess memory while the main features of immune response involve memory. It is an attempt to incorporate fractional order into the mathematical model of HIV-immune system dynamics and it is still an interesting exercise to determine, mathematically, how the order of a fractional differential system affects the dynamics of system.

In the case of one-virus model, the fractional order system has an interior equilibrium under some restriction. By using stability analysis on fractional order system, we obtain sufficient condition on the parameters for the stability of the interior equilibrium. Our analysis shows that the interior equilibrium which is unstable in the classical integer order model can become asymptotically stable in our fractional order model. Note that the interior equilibrium is globally asymptotically stable (GAS) (see [24]) if the terms associated with immune reactions are given by $c Z_{1} I_{1}$ and $b Z_{1} I_{1}$ instead of $c Z_{1} I_{1} /\left(T+I_{1}\right)$ and $b Z_{1} I_{1} /\left(T+I_{1}\right)$ in (3.1). That is, the interior equilibrium of the one-virus model can become unstable because of the frequency dependence (see [4]). However, in our fractional order model with the frequency dependence, the interior equilibrium can also become asymptotically stable if the order $\alpha<0.9916$.

We then consider viral diversity. If the terms associated with immune reaction depend on the mass action law instead of frequency, an interior equilibrium in [24] is GAS. Similar to the integer order model in [4], we find that strange chaotic attractors can be obtained under fractional order model with frequency dependence. That is, the effect of viral diversity and the frequency dependence results in collapse of the immune system and make the behavior of the system dynamics complex. However the chaotic motion may disappear and the fractional order system stabilizes to a fixed point if the value of the order $\alpha$ decreases. The specific biological meaning is deserved to further study.

\section{Acknowledgments}

The authors are very grateful to the referees for their valuable suggestions, which helped to improve the paper significantly. This work was supported in part by Specialized Research Fund for the Doctoral Program of Higher Education from Ministry of Education of China (no. 20060255006), Project of the Shanghai Committee of Science and Technology 
(no. 08JC1400100), and the Open Fund from the Key Laboratory of MICCAI of Shanghai (06dz22103).

\section{References}

[1] M. A. Nowak, R. M. May, and K. Sigmund, "Immune responses against multiple epitopes," Journal of Theoretical Biology, vol. 175, no. 3, pp. 325-353, 1995.

[2] M. A. Nowak and C. R. M. Bangham, "Population dynamics of immune responses to persistent viruses," Science, vol. 272, no. 5258, pp. 74-79, 1996.

[3] M. A. Nowak and R. M. May, Virus Dynamics: Mathematical Principles of Immunology and Virology, Oxford University Press, Oxford, UK, 2000.

[4] S. Iwami, S. Nakaoka, and Y. Takeuchi, "Frequency dependence and viral diversity imply chaos in an HIV model," Physica D, vol. 223, no. 2, pp. 222-228, 2006.

[5] E. Ahmed and A. S. Elgazzar, "On fractional order differential equations model for nonlocal epidemics," Physica A, vol. 379, no. 2, pp. 607-614, 2007.

[6] E. Ahmed, A. M. A. El-Sayed, and H. A. A. El-Saka, "Equilibrium points, stability and numerical solutions of fractional-order predator-prey and rabies models," Journal of Mathematical Analysis and Applications, vol. 325, no. 1, pp. 542-553, 2007.

[7] M. P. Lazarević, "Finite time stability analysis of $\mathrm{PD}^{\alpha}$ fractional control of robotic time-delay systems," Mechanics Research Communications, vol. 33, no. 2, pp. 269-279, 2006.

[8] T. J. Anastasio, "The fractional-order dynamics of brainstem vestibulo-oculomotor neurons," Biological Cybernetics, vol. 72, no. 1, pp. 69-79, 1994.

[9] T. T. Hartley, C. F. Lorenzo, and H. K. Qammer, "Chaos in a fractional order Chua's system," IEEE Transactions on Circuits and Systems I, vol. 42, no. 8, pp. 485-490, 1995.

[10] B. Bonilla, M. Rivero, L. Rodríguez-Germá, and J. J. Trujillo, "Fractional differential equations as alternative models to nonlinear differential equations," Applied Mathematics and Computation, vol. 187, no. 1, pp. 79-88, 2007.

[11] A. M. A. El-Sayed, A. E. M. El-Mesiry, and H. A. A. El-Saka, "On the fractional-order logistic equation," Applied Mathematics Letters, vol. 20, no. 7, pp. 817-823, 2007.

[12] R. Hilfer, Ed., Applications of Fractional Calculus in Physics, World Scientific, River Edge, NJ, USA, 2000.

[13] K. S. Cole, "Electric conductance of biological systems," in Proceedings of the Cold Spring Harbor Symposia on Quantitative Biology, pp. 107-116, Cold Spring Harbor, NY, USA, January 1993.

[14] I. Podlubny, Fractional Differential Equations, vol. 198 of Mathematics in Science and Engineering, Academic Press, San Diego, Calif, USA, 1999.

[15] Z. M. Odibat and N. T. Shawagfeh, "Generalized Taylor's formula," Applied Mathematics and Computation, vol. 186, no. 1, pp. 286-293, 2007.

[16] A. A. Kilbas, H. M. Srivastava, and J. J. Trujillo, Theory and Applications of Fractional Differential Equations, vol. 204 of North-Holland Mathematics Studies, Elsevier, Amsterdam, The Netherlands, 2006.

[17] D. Matignon, "Stability results for fractional differential equations with applications to control processing," in Computational Engineering in Systems Applications, vol. 2, pp. 963-968, IMACS IEEESMC, Lille, France, 1996.

[18] J. Velasco-Hernández, J. García, and D. Kirschner, "Remarks on modeling host-viral dynamics and treatment," in Mathematical Approaches for Emerging and Reemerging Infectious Diseases: An Introduction to Models, Methods, and Theory, vol. 125 of The IMA Volumes in Mathematics and Its Applications, pp. 287-308, Springer, New York, NY, USA, 2002.

[19] R. R. Regoes, D. Wodarz, and M. A. Nowak, "Virus dynamics: the effect of target cell limitation and immune responses on virus evolution," Journal of Theoretical Biology, vol. 191, no. 4, pp. 451-462, 1998.

[20] E. Ahmed, A. M. A. El-Sayed, and H. A. A. El-Saka, "On some Routh-Hurwitz conditions for fractional order differential equations and their applications in Lorenz, Rössler, Chua and Chen systems," Physics Letters A, vol. 358, no. 1, pp. 1-4, 2006.

[21] A. S. Perelson, D. E. Kirschner, and R. De Boer, "Dynamics of HIV infection of CD4 ${ }^{+}$T cells," Mathematical Biosciences, vol. 114, no. 1, pp. 81-125, 1993.

[22] K. Diethelm, N. J. Ford, and A. D. Freed, "A predictor-corrector approach for the numerical solution of fractional differential equations," Nonlinear Dynamics, vol. 29, no. 1-4, pp. 3-22, 2002.

[23] K. Diethelm, N. J. Ford, and A. D. Freed, "Detailed error analysis for a fractional Adams method," Numerical Algorithms, vol. 36, no. 1, pp. 31-52, 2004.

[24] Y. Iwasa, F. Michor, and M. Nowak, "Some basic properties of immune selection," Journal of Theoretical Biology, vol. 229, no. 2, pp. 179-188, 2004. 


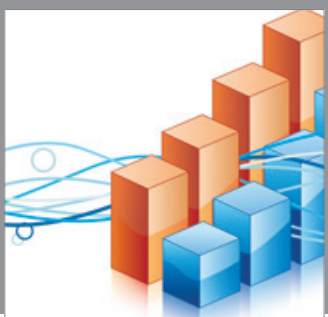

Advances in

Operations Research

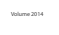

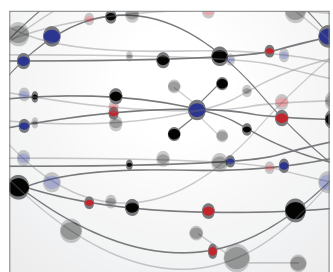

\section{The Scientific} World Journal
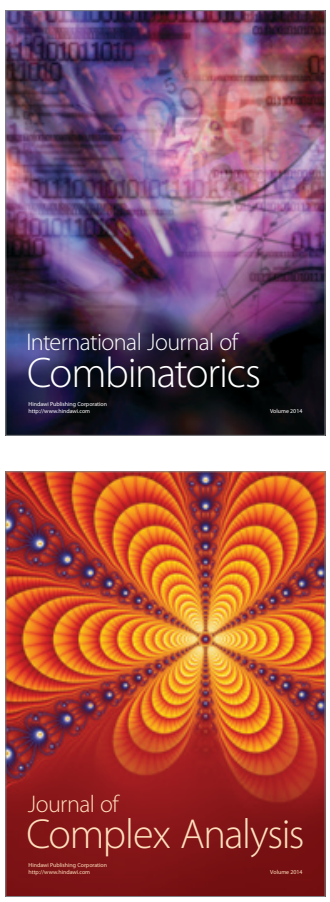

International Journal of

Mathematics and

Mathematical

Sciences
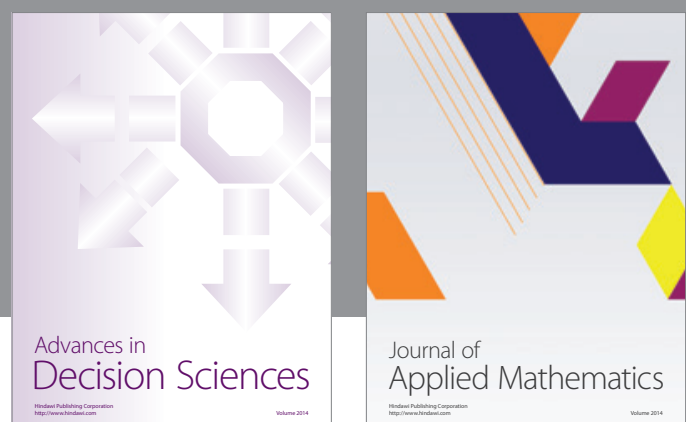

Journal of

Applied Mathematics
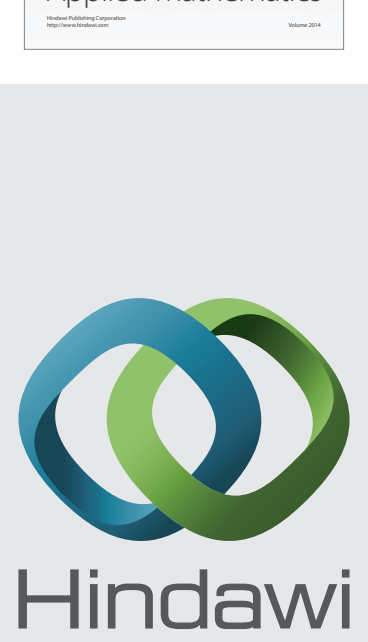

Submit your manuscripts at http://www.hindawi.com
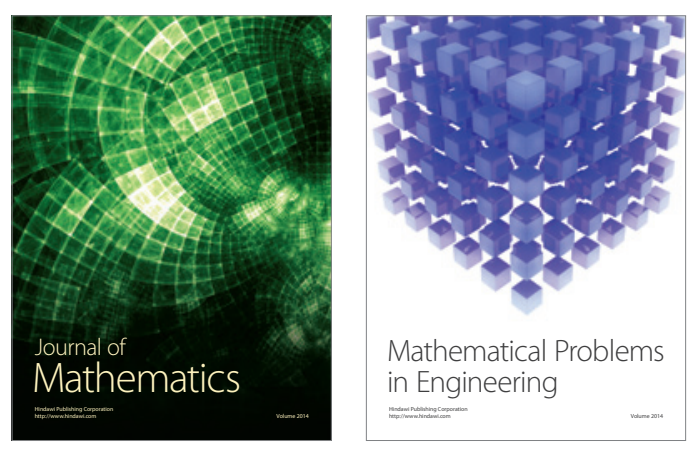

Mathematical Problems in Engineering
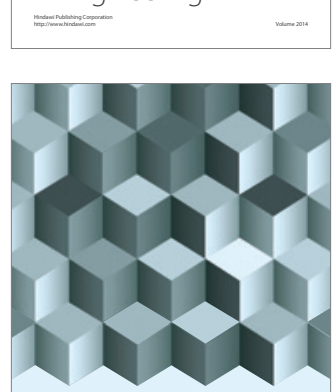

Journal of

Function Spaces
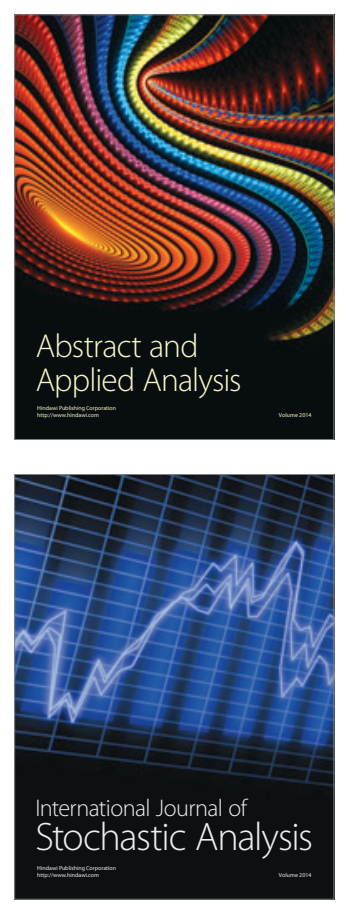

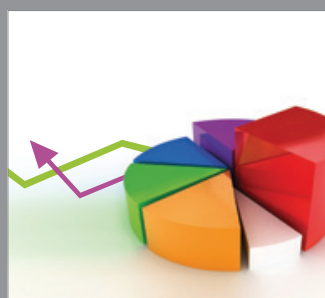

ournal of

Probability and Statistics

Promensencen
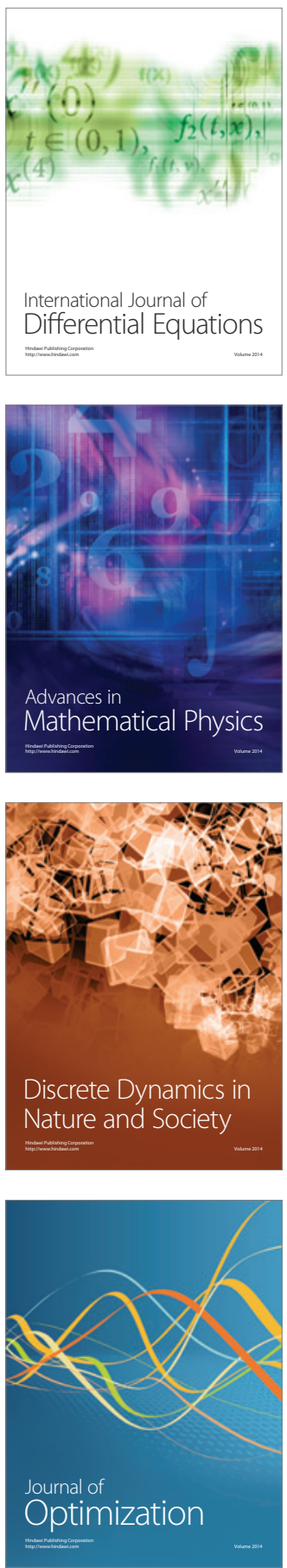